\title{
Real Time Multiple Face Detection Algorithm based on Minimum Facial Features
}

\author{
Mohit Jain \\ Assistant professor \\ KEC Ghaziabad.
}

\author{
Kishore Sethy \\ Project Manager \\ Tech Mahindra
}

\author{
Gaurav Singh \\ Assistant Professor \\ KEC ,Ghaziabad
}

\begin{abstract}
In this paper, we have used the knowledge-based approach to detect the multiple human face of an image. We presented an algorithm, which detects human face by the geometric correlations between location of face and hairs in an image. Range of skin color are used to figure out possible face regions so as to initially localize the face, furthermore, the probable hair blocks in an image frame are determined by means of hair color spectrums. Combined skin and hair blocks decide candidate face areas in light of the geometric relation then we separate each component on the basis of pixel and count the number of faces detected therefore, the proposed is able to be expectedly transplanted to an embedded system, like the developing pet robot so as to perform dynamic face detection and tracking. The algorithm can be used for surveillance. The algorithm can be used for developing secure PC camera and web camera. The algorithm is being used for providing laptop security.
\end{abstract}

\section{General Terms}

Image Processing, algorithms.

\section{Keywords}

Skin Quantization, Hair Quantization

\section{INTRODUCTION}

Human face detection have been importantly developed recently - surveillance systems, digital monitoring, intelligent robots, notebook, PC cameras, digital cameras, 3G cell phones, and the like. These applications accordingly play an important role in our day to day life. However, the algorithms of the applications are quite difficult to analyze and hard to meet real-time requirements of specific framerate.

Over the last few decade much research has been done for improving the performance of human face detection. Detection method are categorized into many types (1)Cascade Object Detector to detect the location of a face in a video frame. The cascade object detector uses the ViolaJones[15] detection algorithm and a trained classification model for detection. By default, the detector is configured to detect faces, but it can be configured for other object types. (2) Knowledge-based method: This method is directed at finding constant features of a face within a complex environment, thereby localizing the position of the face. Relationships among the features helpfully determine whether a human face appears in an image or not [1]. (3)Feature invariant approaches: Invariant features, unresponsive to different positions, brightness, and viewpoints, are utilized in this approach to detect human faces. A statistical model is usually built up for describing the relations among face features and the presence of the detected faces. Such face features, for instance, are Facial Features [2], Texture [3], and Skin Color [4]. (4)Template matching method: A template cohering with human face features is used to perform a pattern-matching operation based on the template Model [6] are common examples of this method. (5)Appearance-based method: This method, such as Eigen face [7], Neural Network [8], and Hidden Markov Model [9], employs a series of face images to train and establish a face model for the face detection. In general, method (1), (3),(4) and (5) are more complex than method (2); yet the more features are used in method (1), the more complicated it is.

We present an complexity reduce algorithm which is designed for detecting human faces in real-time as proposed. This algorithm, on the basis of Knowledge-based method with minimum features, follows the geometric characteristics of skin and hair color to detect human faces.

\section{PROBLEM STATEMENT}

Face detection is a critical prior step that localizes the region of the face in video frames, from which a relevant feature set can be extracted and subsequently served as input to the face recognizer. As such, the accuracy of detection directly impacts the ability to recognize subjects in video.

\section{APPROACH}

Our approach describe that the algorithm consists of main modules which are as follows Face Detection .

Detailed descriptions of each module are as follows:

\subsection{Face Detection}

Face Detection is achieved by using Algorithm Based on Minimum Facial Features. The Steps of the Algorithm are as follows: Taking color information we have to extract features of skin color and hair from a complex background. The flowchart of the proposed algorithm is shown as Figure 3:Face detection modules again separated in to five modules 1(a)Skin Detection: Using color information to detect possible skin color in an image ,1(b) Hair Detection: Utilizing brightness to out where hair probably is1(c) Skin Quantization: Quantizing skin color pixels and identifying blocks of the skin 1(d)Hair Quantization: Quantizing hair color pixels and identifying blocks of the hair 1(e) Get the Fusion of Features: Determining whether the detected skin area is a part of a human face according to the relative positions between skin and hair regions. 


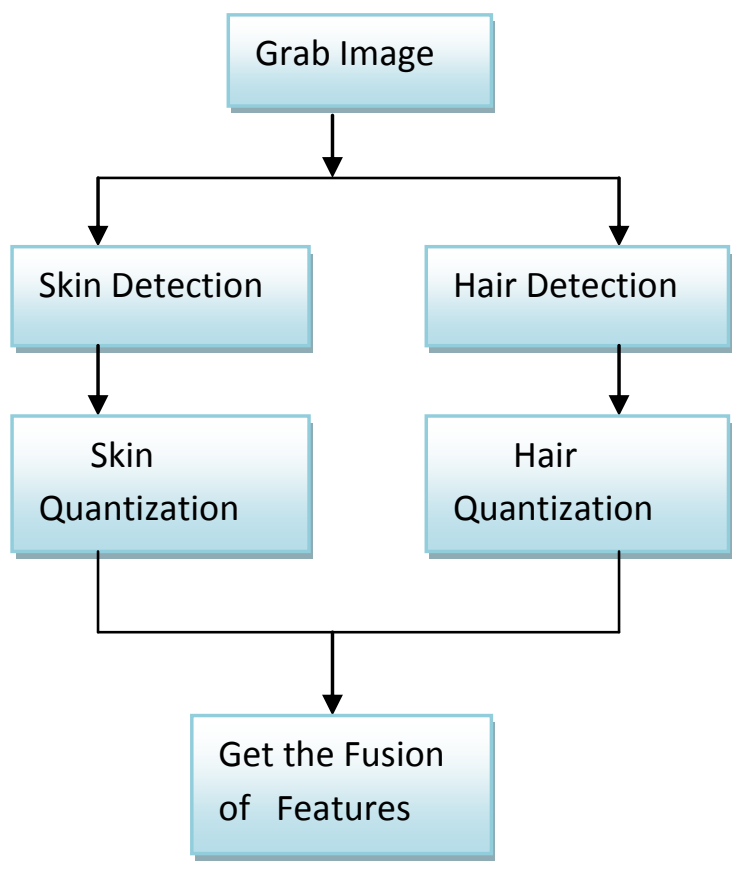

Fig 1: An overview flowchart of Face Detection Algorithm

\subsection{Skin Detection}

There are many studies have been involved in describing the range of skin colour in an model without normalization was sensitive to variations of light, so that The RGB model was therefore transformed to the Normalized RGB model. The formulas for the transformation are listed as equation 1 and 2:

$$
\begin{aligned}
& r=\frac{R}{R+G+B} \\
& g=\frac{G}{R+G+B}
\end{aligned}
$$

Using these two equation the RGB model transferred to normalized RGB model .Equation (1) represents the normalization of a red pixel while equation (2) stands for that of a green pixel.

These two equations declared above accordingly specify the upper limit $F_{1}(r)$ and lower limit $F_{2}(\mathrm{r})$ of the skin color (through analyses of experiments) [11]:

$$
\begin{aligned}
& F_{1}(r)=-1.376 r^{2}+1.0743 r+0.2 \\
& F_{2}(r)=-o .776 r^{2}+0.5601 r+0.18
\end{aligned}
$$

White colour $(r=0.33$ and $g=0.33)$ is also in the defined range, so the following condition is added for excluding the white colour:

$$
w=(r-0.33)^{2}+(g-0.33)^{2}>0.001
$$

Together equation (3),(4) and (5), the skin color range specified

$$
\text { Skin }=\left\{\begin{array}{c}
\left(i f\left(g<F_{1}(r) \cap g>F_{2}(r) \cap w>0.001\right)\right. \\
0 \text { otherwise }
\end{array}\right.
$$

Here is another hues element of the robust HSI colour model[12][13] is taken as a new measure for approximating skin colour pixels so as to improve the above insufficiency. The following exemplify the relation between the RGB colour model and the HSI colour model.

$$
\begin{gathered}
\theta=\cos ^{-1}\left\{\frac{0.5[(R-G)+(R-B)]}{\sqrt{(R-G)^{2}+(R-B)(G-B)}}\right\} \\
\left\{\begin{array}{c}
H=\theta \text { if } B \leq G \\
H=360^{\circ}-\theta \text { if } B>G
\end{array}\right.
\end{gathered}
$$

Modified skin detection condition after experiment equation (8) shows

$$
\text { Skin }=\left\{\begin{array}{l}
1 \text { if }\left(\begin{array}{l}
g<F_{1}(r) \cap g>F_{2}(r) \cap w>0.001 \cap \\
(H>240 \cup H \leq 20)
\end{array}\right) \\
0 \text { otherwise }
\end{array}\right)
$$

Figure 2 is the result of skin dtection of the image dealt with by the equation 8 .

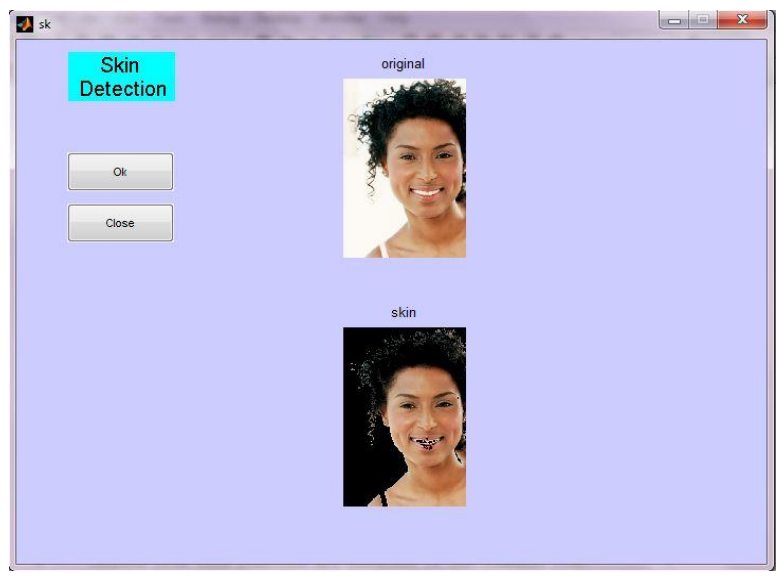

Fig 2: Result after skin detection

\subsection{Hair Detection}

The intensity element of HSI colour model is used for detecting the hair colour. The relation between the intensity element and RGB elements is as follows:

This intensity element is available for measuring the brightness of an image; moreover, the hair colour is specified

$$
I=\frac{1}{3}(\mathrm{R}+\mathrm{G}+\mathrm{B})
$$

to be in the range of dark. Equation 10 specify the intensity range of the hair colour based on experiments.

$$
\text { Hair }=\left\{\begin{array}{l}
1 \text { if }\left\{\begin{array}{l}
(I<80 \cap(B-G<15 \cup B-R<15)) \\
\cup \\
(20<H \leq 40)
\end{array}\right. \\
0 \text { otherwise }
\end{array}\right.
$$

The condition ( $\mathrm{B}-\mathrm{G}<15 \cup \mathrm{B}-\mathrm{R}<15)$ closes out the pixels which are easily categorized as deep blue while hair colour pixels commonly and statistically match the condition $(\mathrm{B}-\mathrm{G}<15 \cup \mathrm{B}-\mathrm{R}<15)$. The condition $(\mathrm{I}<80)$ includes the pixels which are dark. The condition $(20<\mathrm{H} \leq 40)$ includes the pixels which are brown. Figure 6 shows that many nonhair pixels are misjudged, yet the Hair Quantization module 
perform the follow-up operations of the whole algorithm so as to minimize the impact from the misjudging.

Figure 3 shows image processed by the equation 10 . Non-hair color pixels are replaced by white pixel.

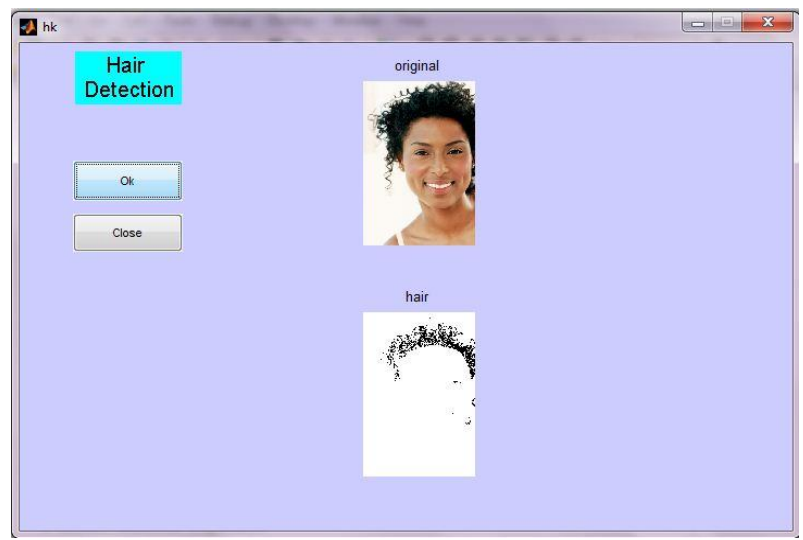

Fig 3: Result after Hair detection

\subsection{Skin Quantization}

This module quantizes skin color pixels and uses a $3 \times 3$ pixel square to express whether or not a region included pixels of skin color. The quantization lowers the image resolution; nonetheless, the following calculation of the geometric locations of pixels is speeded up. The number of black pixels within 9 pixels is counted and these 9 pixels regarded as nonskin color blocks if the number is beyond a threshold value 4 . The rest are viewed as skin color blocks. demonstrates the image of after the Skin Quantization. Figure 4 ,Skin quantization demonstrates the image of Figure 2.

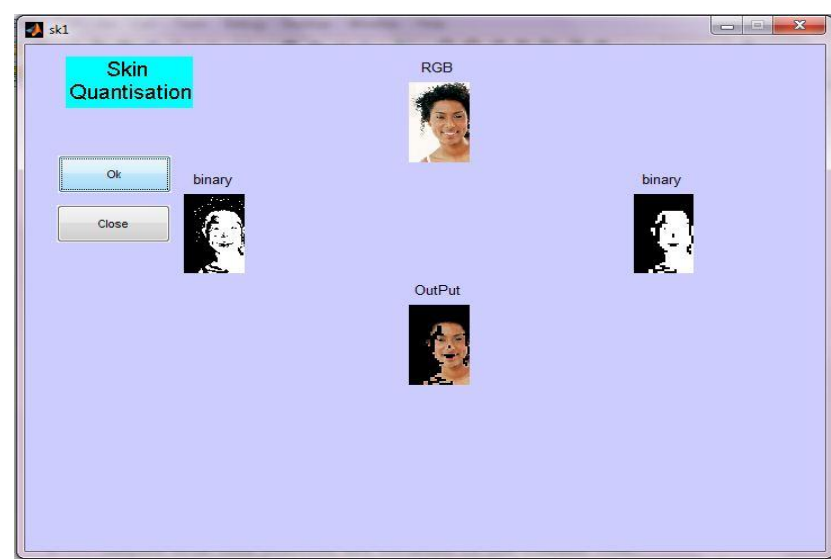

Fig 4: Skin Quantization

\subsection{Hair Quantization}

This module, similar to the Skin Quantization, quantizes hair color pixels and uses a $3 \times 3$ pixel square to express whether or not a region included pixels of hair color. The number of white pixels within 9 pixels is counted and these 9 pixels considered as non-hair color blocks if the number is beyond a threshold value 4 . The rest are treated as hair color blocks.

Figure 5 displays the image of Figure 3 processed by the Hair Quantization.

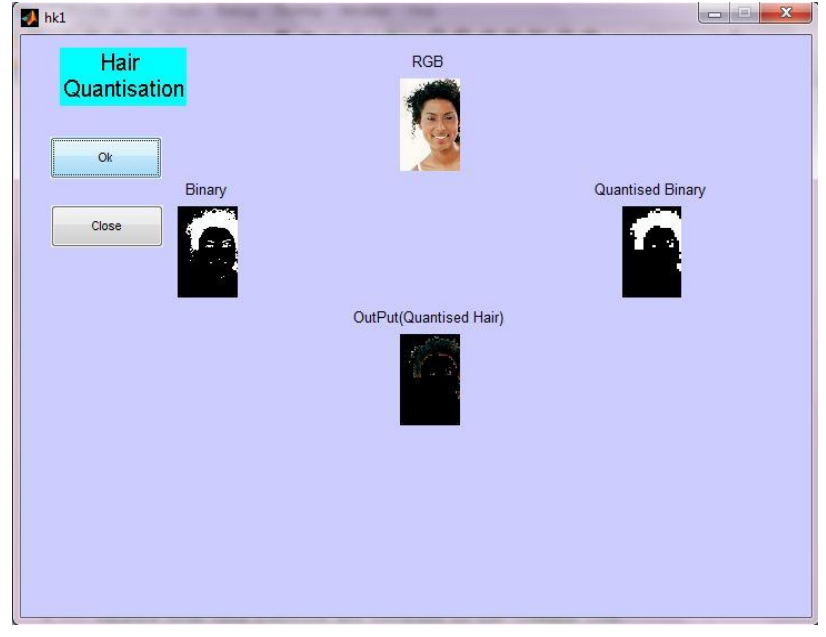

Fig 5: Result of Hair Quantaization

\subsection{Get the Fusion of Features:}

This module, referring to the results from Skin Quantization, and the result from Hair Quantization and determines the existence of a human face as well as its relation. Figure 6 illustrate the flowchart of the human face detection

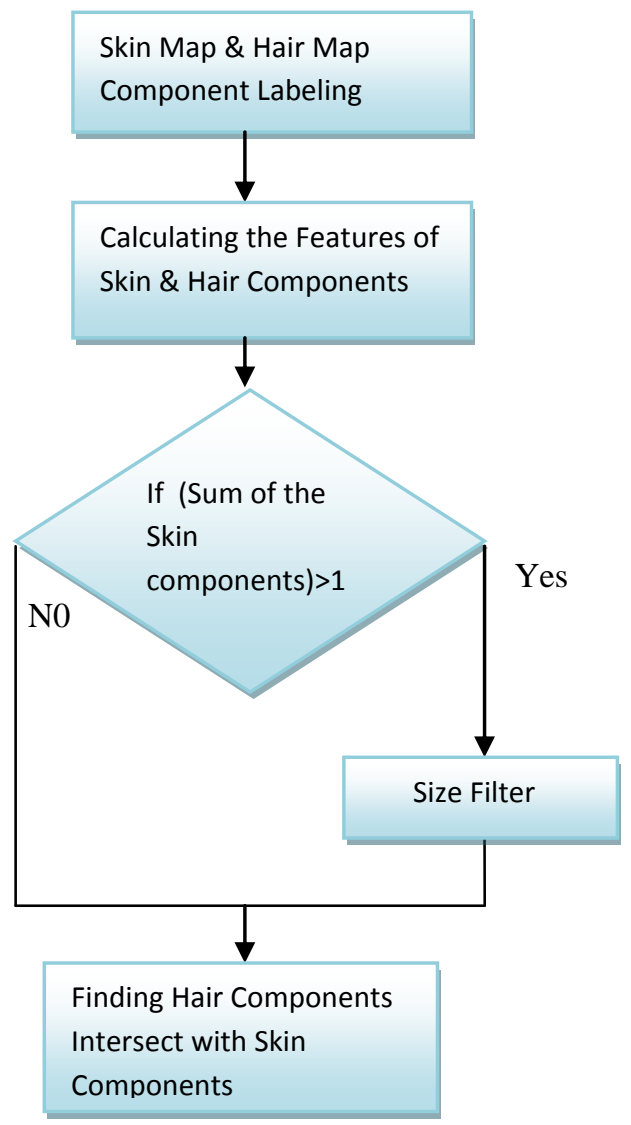

Fig 6: Flowchart of single human face detection

The result from Skin Quantization and Hair Quantization is further calculated using Component Labeling[14]. Adjacent pixels are defined as one component; two components have no adjoining pixels on the boundary. Component Labeling, applying 8 connectivity method, is able to search out all components in an image and get each of them labeled.. Calculating the Features of Skin \& Hair Components: 
The detection algorithm in Figure 6 calculates the features for all currently labeled components. For a component, features to be calculated are area, center of gravity (COG), and the coordinates of the extreme left, top, right, and bottom pixels, respectively.

Size Filter[14] is used to filter out noises and to conserve main skin features. The filtering conditions can be changed according to requirements such as the area of a component. To detect a human face, only one component is required. Therefore among the components only the largest component is preserved while the other small components are eliminated.

Finding Hair Components Intersect with Skin Component :

Then we find the hair components that intersect with the skin component. The presence of human face requires that hair components and skin component intersect. Therefore, when there is no hair component intersecting with skin component, there is no human face present in the picture. From practical observations, the most common twelve hair styles that we would like to detect are shown in Figure 7.

To find out whether the skin and hair components are extracted from a human face, we estimate by the joint geometric relationship. Figure 7 shows the geometric relation between a skin component and a hair component
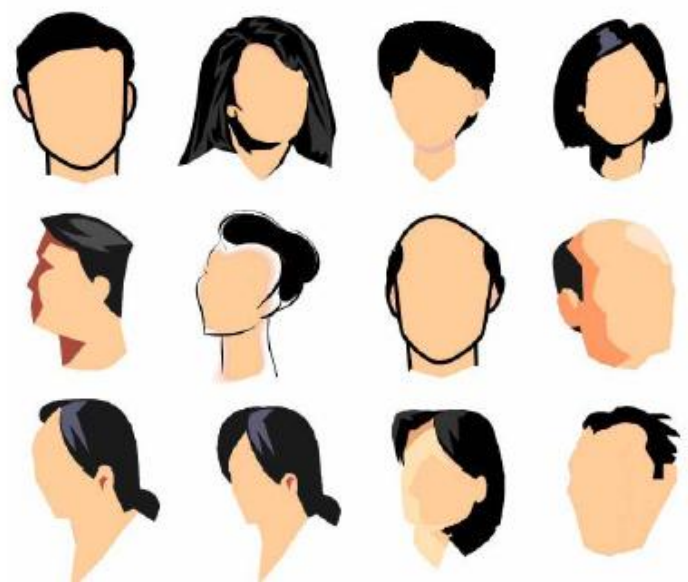

Fig 7 Twelve hair styles like to detect

In order to simplify the twelve complex geometric relations into some simple mathematical operations, we estimate the joint geometric relationship using the "minimum bounding box" approach. The minimum bounding box is calculated for each skin component as well as each hair component. If the minimum bounding box of a skin component intersects with the minimum bounding box of a hair component, they are "related". Observe that the coordinates of each minimum bounding box has already been obtained in the step "calculating the features of Skin and Hair component". The intersection of skin bounding box and hair bounding box is shown in Figure 8.

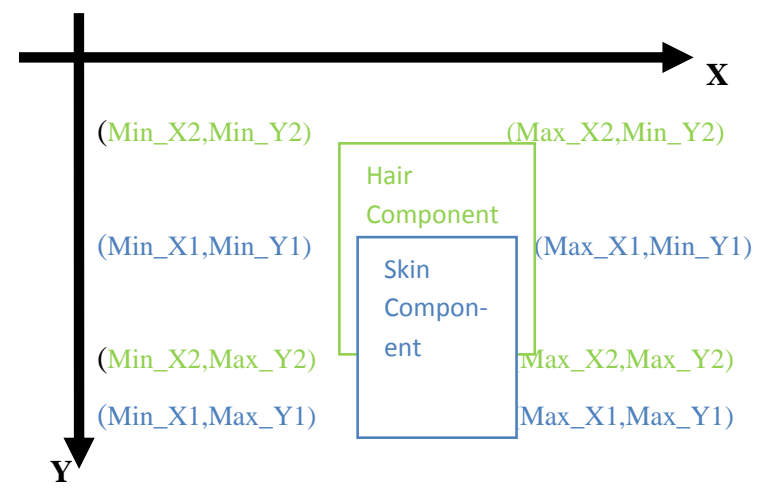

Hair boundary box Skin boundary box

Figure 9 summarizes the various intersection relations between skin and hair bounding boxes of Figure 7 .
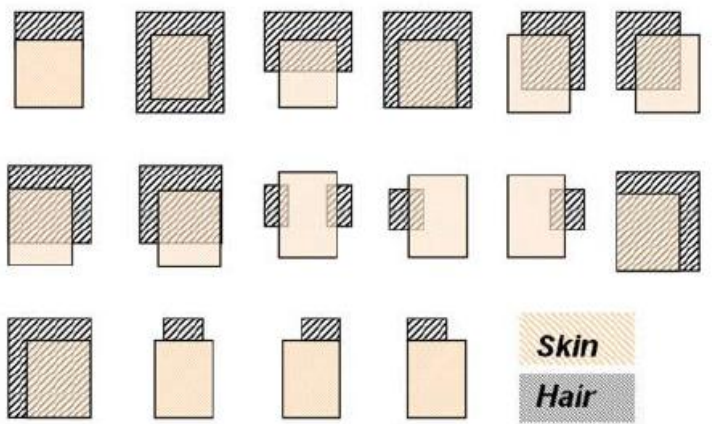

Skin

Hair

Fig 9: Intersection relations between hair and skin bounding boxes.

Observe that not all intersection relationships are possible from a human face. We restrict the intersections to be the ones shown in Figure 9. That is, a hair component is associated with a skin component only if the hair bounding box intersects with skin bounding box according to one of the relations shown in Figure 9. If one hair component is associated with a skin component, the operation for finding hair component is thus finished. Figure 10 shows the result of the face detection

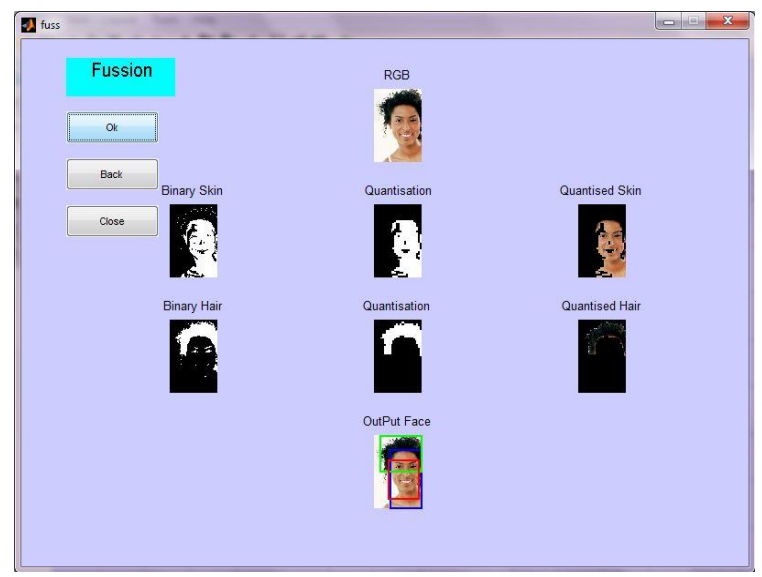

Fig. 10: Result of face detection

\subsection{Multiple Face Detection}

Once human face is being detected using this algorithm, then we separate the component on the basis of number of pixel then we can detect multiple faces from an image also from video file. 


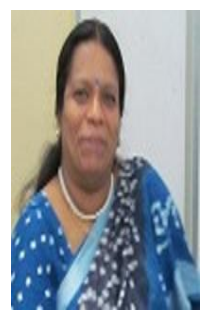

(a)

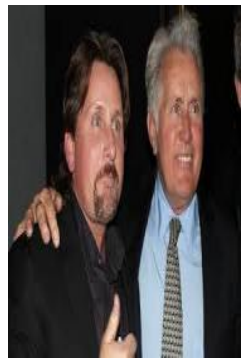

(b)

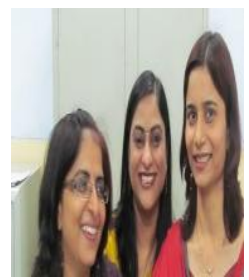

(c)

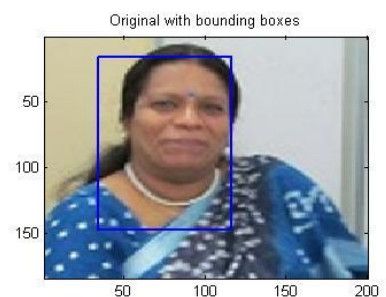

$\mathbf{R}(\mathbf{a})$

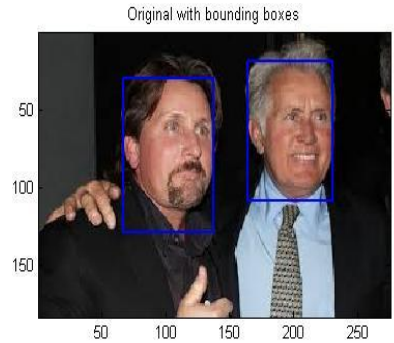

$\mathbf{R}(\mathbf{b})$

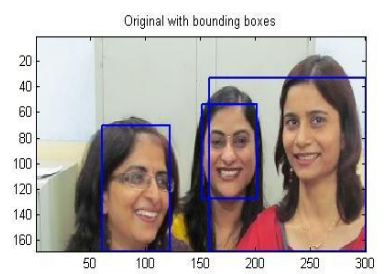

$\mathrm{R}(\mathrm{c})$
Fig 11:(a),(b),(c), R(a),R(b) and R (c)

In this Fig 11 we have taken three image (a),(b) and (c) are original image and after the processing $R(a), R(b)$ and $R(c)$ are result image.

\section{CONCLUSION}

In this paper, we have proposed a more reliable multiple face detection approach based on the geometric relations between skin of human face and hair. The algorithm also takes the advantage of a exact skin color extraction.. The size information can be used to separate into application- and view point dependent classes, for example, large, medium, and small. Similarly, brightness and color information can be used to classify objects into categories such as "bright" or "red.", which can be easily calculated since we know the frame rate of the sequence. . For each such skin bounding box we find the hair bounding box intersecting with it, to determine if a corresponding human face exists.

\section{REFERENCES}

[1] Jerome, M. S.hapiro, 1993. "Embedded Image Coding Using Zerotress of Wavelet Coefficients", IEEE Transaction on Signal Processing Vol.41No.12.

[2] T.K.Leung, M.C.Burl,and P.Perona, 1995“ Finding Face in Cluttered Scenes Using Random Labeled Graph Matching", Fifth IEEE Int'l Conf.Computer Vision, 1995, pp637-644..

[3] Y. Dai and Y.Nakano, 1996 "Face-Texture Model Based on SGLD and Its Application in Face Detection in a Color Scence" , Pattern Recognition,vol. 29,no. 6, 1996, pp.1007-1017.

[4] J. Yang and A.Waibel, 1996 "A Real-Time Face Tracker" , Third Workshop Applications of Computer Vision, 1996,pp. 142-147.

[5] I. Craw, D. Tock, and A. 1992 Bennett, Proc. Second European Conf. Computer Vision, 1992, pp. 92-96. "Finding Face Features,"

[6] A. Lanitis,C.J. Taylor,and T.F. Cootes,. 1995 "An Automatic Face Identification System Using Flexible Appearance Models", Image andVision Computing,vol. 13,no. 5, 1995, pp. 393-401.

[7] M. Turk and A.Pentland, 191 "Eigenface for Recognition, "J.Cognitive Neuroscience,vol.3, 1991, pp.71-86.

[8] H. Rowley,S. Baluja,and T. Kanade, 1998 "Neural Network-Based Face Detection,'IEEE Trans. Pattern Analysis and Matchine Intelligence,vol.20,no.1,Jan. 1998.

[9] A. Rajagopalan,K. Kumar,J.Karlekar,R. Manivasakan,M. Patil,U. Desai, P. Poonacha, and S. Chaudhuri, 1998 "Finding Faces in Photographs" ,Proc. Sixth IEEE Int, 1 Conf. Computer Vision.

[10] Linda G. Shapiro, George C. Stockman ， 2001 “Computer Vision”, Prentice Hall , pp.192-193.

[11] M. Soriano, S. Huovinen, B. Martinkauppi, and M. Laaksonen, 2000"Using the Skin Locus to Cope with Changing Illumination Conditions in Color-Based Face Tracking," Proc. of IEEE Nordic Signal Processing Symposium, pp. 383-386.

[12] Rafael C. Gonzalez, Richard E. Woods, 2002 "Digital Image Processing”, $2^{\text {nd }}$ Edition, Prentice Hall , pp.299300 .

[13] William K. Pratt, 2001 "Digital Image Processing", 3rd Edition, John Willey \& Sons , pp.63-87.

[14] Ramesh Jain, Rangachar Kasturi, Brian G. Schunck, 1995 “Machine Vision”, McGraw-Hill ,pp.44-48.

[15] Viola, Paul A. and Jones, Michael J ,2001 "Rapid Object Detection using a Boosted Cascade of Simple Features", IEEE CVPR.

[16] Yao-Jiunn Chen and Yen-Chun Lin, 2007 "Simple Facedetection Algorithm Based onMinimum Facial Features", IEEE Industrial Electronics Society (IECON) Nov. 5-8.

[17] Oge Marques,2011 “ Practical Image and Video Processing using MATLAB”, A John Wiley \& Sons Inc Publication, pp.561-578. 